Quim. Nova, Vol. 27, No. 1, 62-65, 2004

\title{
TRITERPENOS E FERULATOS DE ALQUILA DE Maprounea guianensis
}

\author{
Juceni P. David* \\ Faculdade de Farmácia, Universidade Federal da Bahia, 40170-290 Salvador - BA \\ Marilena Meira e Jorge M. David \\ Instituto de Química, Universidade Federal da Bahia, 40170-290 Salvador - BA \\ Maria Lenise da S. Guedes \\ Instituto de Biologia, Universidade Federal da Bahia, 40170-290 Salvador - BA
}

Recebido em 20/3/03; aceito em 13/6/03

\begin{abstract}
TRITERPENES AND ALKYL FERULATES FROM MAPROUNEA. This work describes the phytochemical study of hexane extracts from the stem of Maprounea guianensis. Besides 3-oxo-21 $\alpha-H$-hop-22(29)-en (moretenone), $\beta$-sitosterol, lupenone and lupeol, a mixture of dodecosyl, tetracosyl, hexacosyl, octacosyl and triacontyl ferulates was also isolated, as well as $3-\beta$-acetoxy-

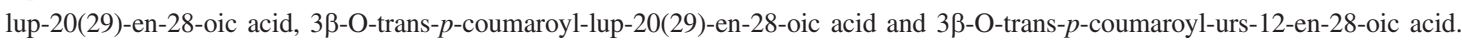
The structures of these compounds were established by spectroscopic analysis.
\end{abstract}

Keywords: Maprounea guianensis; triterpenes; alkyl ferulates.

\section{INTRODUÇÃO}

Maprounea guianensis Aublet é uma árvore pertencente à família Euphorbiaceae, que consiste de 290 gêneros e 7500 espécies distribuídas nas regiões tropicais e temperadas do globo, concentrandose principalmente na África e América tropical ${ }^{1}$. A família Euphorbiaceae consiste de plantas dicotiledôneas, ervas, arbustos e árvores, algumas suculentas semelhantes a cactos, que são caracterizadas freqüientemente pela ocorrência de seiva leitosa geralmente tóxica ${ }^{1}$. Entre as espécies mais conhecidas desta família encontramse quebra-pedra, aveloz, mandioca, mamona e seringueira ${ }^{2}$.

$\mathrm{O}$ estudo do gênero Maprounea tem despertado o interesse dos pesquisadores devido às atividades biológicas de suas espécies. Por exemplo, tem sido atribuída potente atividade anti-HIV e anti-tumoral à espécie $M$. africana ${ }^{3}$. Este gênero é pouco representado no Brasil, não se podendo afirmar se este contribui com uma ou duas espécies no país, haja vista que a identificação botânica da espécie $M$. guianensis tem levantado controvérsias, sendo que alguns autores a identificam como sendo M. brasiliensi $^{4}$. Deste modo, o estudo fitoquímico com as espécies do gênero tem grande importância quimiossistemática. $\mathrm{O}$ estudo anteriormente realizado com o caule de $M$. guianensis coletada em Demerara - Georgetown (Guiana) relata o isolamento de 3-oxo-21 $\beta$ - $H$-hop-22(29)-eno (moretenona) e ácido 3-acetilaleuritólico ${ }^{5}$. Este trabalho descreve o isolamento e elucidação estrutural de metabólitos secundários presentes no extrato hexânico do caule desta espécie, em comparação com os resultados previamente reportados. Deste extrato, além de 3-oxo-21 $\alpha-H$ hop-22(29)-eno ou moretenona (1), $\beta$-sitosterol, lupenona e lupeol foi isolada a mistura dos $E$-ferulatos de dodecosila (2), tetracosila (3), hexacosila (4), octacosila (5), triacontila (6), bem como os ácidos 3- $\beta$-acetoxi-lup-20(29)-eno-28-óico (7), 33-O-trans- $p$-cumaroillup-20(29)-eno-28-óico (8) e 3 $\beta$-O-trans-p-cumaroil-urs-12-eno-28óico (9). A identificação estrutural destas substâncias foi realizada por meio da análise de dados espectrométricos de EMIE e RMN de ${ }^{1} \mathrm{H}$ e ${ }^{13} \mathrm{C}$ (PND e DEPT).

\footnotetext{
*e-mail: juceni@ufba.br
}

\section{PARTE EXPERIMENTAL}

Os espectros de RMN foram obtidos em espectrômetro Gemini 300 da Varian, sendo utilizado o sinal do solvente como referencial interno. Os espectros de massas foram obtidos por meio de injeção direta no detetor de massas da HP modelo 5973.

Os caules de Maprounea guianensis foram coletados na restinga do Parque Metropolitano da Lagoa do Abaeté (Salvador, BA). A identificação da espécie foi realizada e uma exsicata desta foi depositada sob número 041211 no Herbário Alexandre Leal Costa, do Instituto de Biologia da UFBA. Para avaliação da citotoxicidade, tanto dos extratos brutos quanto das substâncias isoladas, foi utilizado o teste de letalidade de Artemia salina ${ }^{6}$.

\section{Isolamento dos constituintes}

Os caules foram secos e moídos, sendo a seguir macerados com $\mathrm{MeOH}$. O extrato metanólico do caule foi então submetido a sucessivas partições entre hexano: $\mathrm{MeOH} / \mathrm{H}_{2} \mathrm{O}(9: 1), \mathrm{CHCl}_{3}: \mathrm{MeOH} / \mathrm{H}_{2} \mathrm{O}$ (6:4) e AcOEt: $\mathrm{H}_{2} \mathrm{O}$ (6:4).

O extrato hexânico obtido $(8,68 \mathrm{~g})$ foi submetido a $\mathrm{CC}$ de sílica gel, usando como sistema de solvente hexano:AcOEt em ordem crescente de polaridade e as frações obtidas foram, então, purificadas como descrito a seguir. A fração eluída em hexano:AcOEt (9:1) foi submetida a nova CC sob sílica gel $(40-60 \mu \mathrm{m})$ utilizando como eluente $\mathrm{CHCl}_{3}$ que, a seguir, foi purificada por recristalização em hexano. A parte insolúvel foi identificada como 3-oxo-21 $\alpha$ - $H$-hop-22(29)-eno (1, 265,0 mg). A fração da CC inicial eluída em hexano:AcOEt (8:2) foi submetida a CC sob sílica gel $(40-60 \mu \mathrm{m})$ utilizando como eluentes $\mathrm{CHCl}_{3}$ :AcOEt (99:1). Deste modo, obteve-se o ácido 3- $\beta$-acetoxilup-20(29)-eno-28-óico (7, 11,8 mg). A fração (CC inicial) eluída em hexano:AcOEt (6:4) foi submetida a CC sob sílica gel $(40-60 \mu \mathrm{m})$ utilizando como eluente hexano:AcOEt (7:3), seguida de purificação por CCDP desenvolvida com $\mathrm{CHCl}_{3}$ :AcOEt (95:5), fornecendo o ácido 3ß-O-trans-p-cumaroil-lup-20(29)-eno-28-óico (8, 21,7 mg) e o ácido 3ß-O-trans-p-cumaroil-urs-12-eno-28-óico (9, 27,6 mg). Enquanto que a mistura de ferulatos $(2$ - 6) foi obtida a partir de quatro $\mathrm{CC}$ sucessivas $\mathrm{em} \mathrm{SiO}_{2}$ usando como eluente em cada coluna, 
hexano/AcOEt (6:4), $\mathrm{CHCl}_{3} / \mathrm{AcOEt}$ (99:1), hexano/AcOEt (8:2) e hexano:AcOEt (9:1), respectivamente.

\section{Dados físicos das substâncias isoladas}

3-Oxo-21 $\alpha$ - $H$-hop-22(29)-eno (1), $\mathrm{C}_{30} \mathrm{H}_{48} \mathrm{O}$. EM $\mathrm{m} / \mathrm{z}$ (int. rel.): 424 (24), 205 (29), 189 (100), 190 (22), 191 (13), 149 (12), 148 (14), 147 (18), 123 (14), 121 (22), 120 (8), 119 (17), 109 (20), 108 (9), 107 (27), 105 (17), 95 (30), 93 (28), 91 (16), 69 (20), 68 (15), 67 (25), 55 (28). RMN ${ }^{1} \mathrm{H}\left[300 \mathrm{MHz}, \mathrm{CDCl}_{3}, \delta\right.$ (ppm), multiplicidade, J (Hz)]: $\delta$ 2,41 ( $m, \mathrm{H}-2), 1,05(s, \mathrm{Me}-23), 0,93(s, \mathrm{Me}-24), 1,00(s$, Me-25 e Me-26), 0,91 ( $s, \mathrm{Me}-27), 0,67$ ( $s, \mathrm{Me}-28), 4,65$ ( $d, \mathrm{H}-29 \mathrm{a}$, $\mathrm{J}=1,1 \mathrm{~Hz}), 4,67(d, \mathrm{H}-29 \mathrm{~b}, \mathrm{~J}=1,1 \mathrm{~Hz}), 1,65(s, \mathrm{Me}-30)$. Dados de RMN ${ }^{13} \mathrm{C}$ (Tabela 1).

Mistura dos E-ferulatos de dodecosila (2), tetracosila (3), hexacosila (4), octacosila (5), triacontila (6). EM m/z: $502\left(\mathrm{C}_{32} \mathrm{H}_{54} \mathrm{O}_{4}\right)$, $530\left(\mathrm{C}_{34} \mathrm{H}_{58} \mathrm{O}_{4}\right), 558\left(\mathrm{C}_{36} \mathrm{H}_{62} \mathrm{O}_{4}\right), 586\left(\mathrm{C}_{38} \mathrm{H}_{66} \mathrm{O}_{4}\right)$ e $614\left(\mathrm{C}_{40} \mathrm{H}_{70} \mathrm{O}_{4}\right)$. Dados de RMN ${ }^{1} \mathrm{H} \mathrm{e}{ }^{13} \mathrm{C}$ comparados com os encontrados na literatura ${ }^{7,8} . \mathrm{RMN}^{1} \mathrm{H}\left[300 \mathrm{MHz}, \mathrm{CDCl}_{3}, \mathrm{~d}(\mathrm{ppm})\right.$, multiplicidade, $\left.\mathrm{J}(\mathrm{Hz})\right]$ : $\delta 0,89\left(\underline{\mathrm{H}}_{3} \mathrm{C}, t, 7,0\right), 1,27$ (n-1, $\left.m\right), 1,27$ (H-4'a, n-2, $\left.m\right), 1,59(\mathrm{H}-2$ ' e H-3', $m), 3,94\left(\underline{\mathrm{H}}_{3} \mathrm{CO}, s\right), 4,20$ (H-1', $\left.t, 6,8\right), 6,31(\mathrm{H}-8, d, 15,9)$, 6,93 (H-5, d, 8,2), 7,05 (H-2, $d, 1,8), 7,08$ (H-6, dd, 1,8; 8,1), 7,62 $(\mathrm{H}-7, d, 15,9) . \mathrm{RMN}^{13} \mathrm{C}\left[75 \mathrm{MHz}, \mathrm{CDCl}_{3}, \delta(\mathrm{ppm})\right]: \delta 127,09(\mathrm{C}-1)$, 109,33 (C-2), 147,91 (C-3), 146,76 (C-4), 114,69 (C-5), 123,00 (C6), 144,57 (C-7), 115,74 (C-8), 167,32 (C-9), 55,93 (H $\underline{\mathrm{CO}}$ ), 64,59 (C-1'), 31,91 (C-2'), 25,98 (C-3'), 26,0 - 29,67 (n-3), 31,91 (n-2'), 22,66 (n-1'), 14,07 ( $\left.\mathrm{H}_{3} \underline{\mathrm{C}}^{\prime}\right)$.

Ácido 3- $\beta$-acetoxi-lup-20(29)-eno-28-óico (7), $\mathrm{C}_{32} \mathrm{H}_{50} \mathrm{O}_{4}$. EM m/z (int. rel.): 498 (3), 482 (2), 452 (3), 438 (24), 423 (12), 395 (15), 248 ( 23), 234 (16), 203 (26), 189 (100), 190 (44), 191 (26), 175 (27). $\mathrm{RMN}{ }^{1} \mathrm{H}\left[300 \mathrm{MHz}, \mathrm{CDCl}_{3}, \delta\right.$ (ppm), multiplicidade, J (Hz)]: $\delta$ 4,49 ( $d d, \mathrm{~J}=9,7$ e $5,9 \mathrm{~Hz}, \mathrm{H}-3), 2,06\left(s, \underline{\mathrm{H}}_{3} \mathrm{CCOO}\right), 0,98$ ( $\left.s, \mathrm{Me}-23\right), 0,94(s$, Me-24), 0,86 ( $s, \mathrm{Me}-25), 0,85$ ( $s, \mathrm{Me}-26), 0,84(s, \mathrm{Me}-27), 4,75(d, \mathrm{H}-$ 29a), 4,62 (d, H-29b), 1,71 ( $s, \mathrm{Me}-30)$. Dados de RMN ${ }^{13} \mathrm{C}$ (Tabela 1).

Ácido 3ß-O-trans-p-cumaroil-lup-20(29)-eno-28-óico (8), $\mathrm{C}_{39} \mathrm{H}_{54} \mathrm{O}_{5}$. EM m/z (int. rel.): 602 (2), 438 (14), 439 (6), 395 (12), 248 (33), 203 (20), 191 (22), 190 (30), 189 (38), 187 (18), 175 (13), 164 (20), 163 (13), 147 (100), 136 (13), 135 (12), 133 (17), 121 (27), 120 (10), 119 (23), 107 (18), 93 (16), 91 (14), 83 (11), 81 (17), 71 (10), 69 (22), 57 (14), 55 (21). RMN ${ }^{1} \mathrm{H}\left[300 \mathrm{MHz}, \mathrm{CDCl}_{3}, \delta\right.$ (ppm) , multiplicidade, J (Hz)]: $\delta 7,43$ ( $d$, J=9 Hz, H-2' e H-6'), 6,85 ( $d$, J=9 Hz, H-3' e H-5'), 7,61 ( $d$, J=15,9 Hz, H-7'), 6,30 ( $d$, J=15,9 Hz, H-8'), 4,15 ( $m, \mathrm{H}-3$ ), 4,75 ( $s l, \mathrm{H}-29 \mathrm{a}), 4,62$ ( $s l, \mathrm{H}-29 \mathrm{~b}), 1,72$ ( $s$, Me-30), 0,99 ( $s$, Me-23), 0,95 ( $s$, Me-24), 0,92 ( $s$, Me-25), 0,89 ( $s$, Me-26), 0,88 ( $s, \mathrm{Me}-27)$. Dados de RMN ${ }^{13} \mathrm{C}$ (Tabela 1).

Ácido 3ß-O-trans-p-cumaroil-urs-12-eno-28-óico (9). $\mathrm{C}_{39} \mathrm{H}_{54} \mathrm{O}_{5}$. EM m/z (int. rel.): 602 (2), 438 (6), 395 (1), 248 (100), 249 (20), 219 (13), 203 (82), 204 (25), 205 (11), 192 (15), 191 (81), 190 (89), 189 (44), 187 (7), 188 (7), 175 (17), 165 (13), 163 (11), 147 (50), 133 (39), 121 (10), 119 (9), 105 (8). RMN ${ }^{1} \mathrm{H}\left[300 \mathrm{MHz}, \mathrm{CDCl}_{3}, \delta\right.$ (ppm), multiplicidade, J (Hz)]: $\delta$ 7,39 ( $d$, J=9 Hz, H-2' e H-6'), 6,81 ( $d$, J=9 Hz, H-3' e H-5'), 7,58 ( $d$, J=15,9 Hz, H-7'), 6,26 ( $d$, J=15,9 Hz, H-8'), 4,55 ( $t$, H-3), 5,20 $(t, \mathrm{H}-12), 0,95(s, \mathrm{Me}-23), 0,75$ ( $s, \mathrm{Me}-24), 0,89(s, \mathrm{Me}-25), 0,82(s$, Me-26), 1,05 ( $s$, Me-27). Dados de RMN ${ }^{13} \mathrm{C}$ (Tabela 1).

\section{Teste de citotoxicidade}

Teste de letalidade de Artemia salina. O teste foi realizado a partir do método adaptado por Serrano e colaboradores ${ }^{6}$. A descrição do procedimento experimental detalhado encontra-se na literatura $^{9}$. O extrato hexânico do caule de Maprounea guianensis não apresentou atividade citotóxica (DL > 1000). No entanto, o extrato clorofórmico e acetato de etila apresentaram $90 \%$ e $100 \%$ de ativi- dade, respectivamente. As substâncias 1-9 foram testadas e mostraram-se inativas (DL > 1000).

\section{RESULTADOS E DISCUSSÃO}

O $\beta$-sitosterol, a lupenona e o lupeol foram identificados através da comparação dos dados de RMN com os descritos na literatura ${ }^{8,10}$.

A substância 1 apresentou o pico do íon molecular em $\mathrm{m} / z, 424$ no espectro de massas que, aliado aos dados obtidos dos espectros de $\mathrm{RMN}{ }^{13} \mathrm{C}$ (Tabela 1), permitiu sugerir a fórmula molecular $\mathrm{C}_{30} \mathrm{H}_{48} \mathrm{O}$. Além disso, o pico base registrado em $\mathrm{m} / \mathrm{z}, 189$ indicou que esta subs-

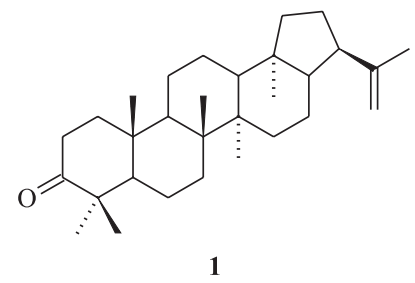

Tabela 1. Dados de RMN ${ }^{13} \mathrm{C}$ das substâncias 1, 7, 8 e 9 [75 MHz, $\delta$ (ppm)]

\begin{tabular}{|c|c|c|c|c|}
\hline Carbono & $\mathbf{1}^{\mathrm{a}}$ & $7^{\mathrm{a}}$ & $8^{\mathrm{a}}$ & $9^{b}$ \\
\hline 1 & 39,57 & 38,40 & 37,06 & 38,28 \\
\hline 2 & 34,15 & 23,69 & 23,83 & 23,21 \\
\hline 3 & 217,88 & 80,96 & 81,01 & 80,86 \\
\hline 4 & 47,40 & 37,80 & 38,07 & 37,85 \\
\hline 5 & 54,86 & 55,43 & 55,42 & 55,27 \\
\hline 6 & 19,76 & 18,17 & 18,17 & 18,15 \\
\hline 7 & 32,67 & 34,25 & 34,22 & 32,94 \\
\hline 8 & 41,60 & 40,71 & 40,73 & 39,43 \\
\hline 9 & 49,73 & 50,41 & 50,35 & 47,41 \\
\hline 10 & 36,80 & 37,13 & 37,17 & 37,85 \\
\hline 11 & 21,59 & 20,87 & 20,85 & 23,59 \\
\hline 12 & 23,92 & 25,46 & 25,42 & 125,37 \\
\hline 13 & 48,83 & 38,40 & 38,43 & 138,10 \\
\hline 14 & 42,32 & 42,42 & 42,45 & 41,99 \\
\hline 15 & 32,67 & 30,57 & 30,56 & 29,57 \\
\hline 16 & 20,83 & 32,16 & 32,15 & 24,13 \\
\hline 17 & 53,87 & 56,37 & 56,40 & 47,80 \\
\hline 18 & 44,18 & 46,94 & 46,95 & 52,74 \\
\hline 19 & 40,19 & 49,27 & 49,25 & 39,00 \\
\hline 20 & 27,33 & 150,36 & 150,34 & 38,82 \\
\hline 21 & 47,85 & 29,69 & 29,69 & 30,58 \\
\hline 22 & 147,97 & 37,04 & 37,06 & 36,74 \\
\hline 23 & 26,58 & 27,95 & 28,02 & 28,02 \\
\hline 24 & 21,10 & 16,49 & 16,65 & 16,82 \\
\hline 25 & 15,74 & 16,17 & 16,19 & 15,40 \\
\hline 26 & 16,50 & 16,06 & 16,13 & 16,77 \\
\hline 27 & 16,50 & 14,65 & 14,67 & 25,74 \\
\hline 28 & 15,17 & 181,50 & 181,74 & 180,05 \\
\hline 29 & 109,50 & 109,71 & 109,73 & 16,91 \\
\hline 30 & 19,67 & 19,34 & 19,35 & 21,04 \\
\hline $\mathrm{H}_{3} \mathrm{CCO}$ & - & 171,04 & - & - \\
\hline $\mathrm{H}_{3} \mathrm{C} C \mathrm{O}$ & - & 21,29 & - & - \\
\hline 1 & - & - & 127,18 & 126,16 \\
\hline $2^{\prime}$ & - & - & 129,90 & 129,80 \\
\hline $3^{\prime}$ & - & - & 116,09 & 115,70 \\
\hline $4^{\prime}$ & - & - & 157,89 & 159,01 \\
\hline 5 & - & - & 116,09 & 115,70 \\
\hline $6^{\prime}$ & - & - & 129,90 & 129,80 \\
\hline $7^{\prime}$ & - & - & 144,15 & 144,48 \\
\hline $8^{\prime}$ & - & - & 115,88 & 115,25 \\
\hline $9^{\prime}$ & - & - & 167,46 & 167,62 \\
\hline
\end{tabular}

a -Solvente $\mathrm{CDCl}_{3}$, b- Solvente $\mathrm{CDCl}_{3}$ e gotas de $\mathrm{CD}_{3} \mathrm{OD}$ 
tância possuía um esqueleto triterpênico pentacíclico da série lupeno ou hopeno ${ }^{11}$. Esta observação foi suportada pela análise dos dados de RMN, uma vez que o espectro RMN ${ }^{1} \mathrm{H}$ mostrou a presença de sete metilas (singletos), destacando-se dentre estes o sinal da metila ligada a carbono $s p^{2}$ em $\delta 1,65$ que, juntamente com os dubletos em $\delta 4,67$ e $\delta 4,65$ atribuídos aos dois hidrogênios olefínicos, caracterizam o grupo isopropenil. Nesse espectro foi também observada a presença de um multipleto em $\delta$ 2,41 atribuído aos dois hidrogênios $\alpha$-carbonílicos. No espectro de $\mathrm{RMN}$ de ${ }^{13} \mathrm{C}$, a presença do sinal em $\delta 217$ e a ausência do sinal do carbono oximetínico $(\delta 79)$ sugeriu a presença de uma carbonila em C-3. Comparação dos deslocamentos químicos encontrados nos espectros $\mathrm{RMN}{ }^{13} \mathrm{C}$ especialmente do $\mathrm{C}$ 17, C-18, C-19, C-21 e do grupo isopropenil da substância 1 com os mesmos carbonos no $21 \alpha H$-hop-22(29)-eno, hopeno e derivados de lupeno permitiu determinar que a substância 1 pertence à série $21 \alpha H$ hop-22(29)-eno ${ }^{12}$. Triterpenos do grupo hopeno podem apresentar o grupo isopropil no C-21 em pseudo-axial e pseudo-equatorial. Estes podem ser diferenciados pelos deslocamentos químicos do C-17, C18, C-19 e especialmente C-21. Nos triterpenos onde o grupo isopropil possui a configuração pseudo-axial, o C-21 é registrado em menor deslocamento químico $(\delta 46,5)$ e naqueles em que este grupo está pseudo-equatorial (série $21 \alpha H$ ), o C-21 encontra-se mais desprotegido ( $\delta 48$ ). Na literatura ${ }^{5,10}$ são encontrados dados de RMN ${ }^{13} \mathrm{C}$ comparáveis com os encontrados para a substância $\mathbf{1}$.

$\mathrm{O}$ espectro RMN de ${ }^{1} \mathrm{H}$ de $\mathbf{2}$ - $\mathbf{6}$ mostrou os sinais característicos do grupo trans-feruloíla. Entre estes, pode-se observar sinais para três hidrogênios aromáticos 1,2,4-tri-substituído, além de dois hidrogênios olefínicos conjugados à carbonila em relação trans $(\mathrm{J}=$ $15,9 \mathrm{~Hz}$ ), bem como a presença do sinal de metoxila ligado a anel aromático $(\delta 3,94)$. A presença da cadeia alcoxílica foi caracterizada pelo tripleto em $\delta 0,89$ referente aos hidrogênios do grupo metila terminal, pelo singleto largo em $\delta 1,27$ correspondente aos hidrogênios metilenos e pelo tripleto desprotegido em $\delta 4,20$ referente aos hidrogênios oximetilênicos. A análise dos espectros de RMN de ${ }^{13} \mathrm{C}$ (Tabela 1) e de massas, bem como comparação com dados da literatura $^{8}$ sugeriu que estas substâncias eram ferulatos de alquilas. Assim, foi determinado o comprimento das cadeias alquílicas dos ferulatos presentes e a proporção entre eles, com base nos valores dos picos do EM e suas abundâncias relativas. Os picos dos íons moleculares registrados em $\mathrm{m} / \mathrm{z}, 614$ (3), $586 \mathrm{~m} / \mathrm{z}$ (357), $\mathrm{m} / \mathrm{z} 558$ (719), $\mathrm{m} / z 530$ (76) e $m / z 502$ (24) no espectro EMIE mostraram que esta mistura era constituída dos $E$-ferulatos de triacontila, $\left(\mathrm{C}_{40} \mathrm{H}_{70} \mathrm{O}_{4}, 2\right)$, octacosila $\left(\mathrm{C}_{38} \mathrm{H}_{66} \mathrm{O}_{4}, 3\right)$, hexacosila $\left(\mathrm{C}_{36} \mathrm{H}_{62} \mathrm{O}_{4}, 4\right)$, tetracosila $\left(\mathrm{C}_{34} \mathrm{H}_{58} \mathrm{O}_{4}, \mathbf{5}\right)$ e dodecosila $\left(\mathrm{C}_{32} \mathrm{H}_{54} \mathrm{O}_{4}, \mathbf{6}\right)$ nas proporções $0,3: 30,3$ : $61,0: 6,4: 2,0$ respectivamente.<smiles>CCCOC(=O)/C=C/c1ccc(O)c(OC)c1</smiles>

$$
\begin{aligned}
& \text { 2: } n=20 \\
& \text { 3: } n=22 \\
& \text { 4: } n=24 \\
& \text { 5: } n=25 \\
& \text { 6: } n=28
\end{aligned}
$$

Os espectros de $\mathrm{RMN}{ }^{1} \mathrm{H}$ de 7 e $\mathbf{8}$ mostraram a presença de sinais característicos de hidrogênios olefínicos entre $\delta 4,7-\delta 4,6$ bem como os duplos dubletos ( $\delta$ 4,49 e 4,15), que foram atribuídos aos hidrogênios oximetínicos, que encontravam-se mais desprotegidos que o esperado na posição 3 dos triterpenos. Este sinal sugere a esterificação de triterpenos nesta posição. Nos espectros de RMN ${ }^{13} \mathrm{C}$ foram verificados os sinais característicos dos carbonos olefínicos dos triterpenos do grupo lupeno em aproximadamente $\delta 150$ (C-20) e $\delta$ 109 (C-29), bem como o sinal do carbono oximetínico (C-3) mais desprotegido que o esperado, devido à esterificação em $c a$. $\delta 80$. O triterpeno 7 apresentou 32 sinais no espectro de $\mathrm{RMN}{ }^{13} \mathrm{C}$ e, juntamente com o pico do íon molecular registrado em $\mathrm{m} / \mathrm{z}, 498$ no espectro EMIE, permitiram sugerir a fórmula molecular $\mathrm{C}_{32} \mathrm{H}_{50} \mathrm{O}_{4}$. Foram ainda observados nos espectros de $\mathrm{RMN}$ de ${ }^{1} \mathrm{H}$ e de ${ }^{13} \mathrm{C}$, o singleto característico de metila de grupo acetoxila $(\delta 2,06)$, além dos sinais dos carbonos carbonílico e metílico de grupo acetoxila em $\delta 171,0$ e 21,3, respectivamente, e o sinal referente ao grupo carboxílico de ácido (C-28) em $\delta$ 181,5. Comparação com os valores de $\mathrm{RMN}{ }^{13} \mathrm{C}$ da literatura empregando dois modelos de triterpenos como referência, o ácido betulínico ${ }^{13}$ e 3- $\beta$-acetiloxi-lup-20-(29)-eno ${ }^{10}$ permitiu atribuir os valores dos deslocamentos químicos dos carbonos e determinar a estrutura da substância 7 como sendo ácido 3- $\beta$-acetiloxilup-20(29)-eno-28-óico.

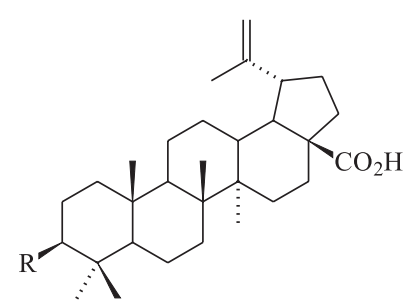<smiles>[R]=CC(=O)OC</smiles><smiles>[R]Oc1ccc(/C=C/C(=O)OC)cc1</smiles>

Os dados obtidos dos espectros de RMN ${ }^{13} \mathrm{C}$ (Tabela 1) e RMN ${ }^{1} \mathrm{H}$ além do pico do íon molecular registrado em $\mathrm{m} / \mathrm{z} 602$ no espectro EMIE sugeriram a fórmula molecular $\mathrm{C}_{39} \mathrm{H}_{54} \mathrm{O}_{5}$ para a substância 8 . $\mathrm{O}$ espectro de $\mathrm{RMN}{ }^{1} \mathrm{H}$ desta substância apresentou também os sinais em $\delta$ 7,61 e $\delta$ 6,30, característicos para o sistema carbonílico $\alpha, \beta$-insaturado com configuração trans $(d, \mathrm{~J}=15,9 \mathrm{~Hz})$. Foi ainda observado o par de dubletos integrado para dois hidrogênios cada em $\delta 7,43$ e $\delta 6,85(\mathrm{~J}=9 \mathrm{~Hz})$ indicativos de anel aromático paradissubstituído. Este conjunto de dados foi sugestivo da esterificação da unidade triterpênica com o ácido $p$-cumárico. Os sinais do espectro de $\mathrm{RMN}{ }^{13} \mathrm{C}$ quando comparados com os valores da literatura confirmaram a presença da unidade $p$-cumaroíla ${ }^{14}$. Esta informação foi corroborada pelo espectro de massas, cujo pico base foi o fragmento em $m / z$ 147, típico da unidade $p$-cumaroíla. No espectro de $\mathrm{RMN}{ }^{13} \mathrm{C}$ além dos sinais da unidade $p$-cumaroíla, pode-se observar a presença da carboxila de ácido na posição C-28, através do sinal em $\delta 181,7$. A comparação com os valores de $\mathrm{RMN}{ }^{13} \mathrm{C}$ da literatura empregando dois modelos de triterpenos como referência, respectivamente o ácido betulínico e 3- $\beta$-acetiloxi-lup-20-(29)-eno ${ }^{10}$ permitiu propor a estrutura da substância 8 como sendo o ácido 3- $\beta$-Otrans-p-cumaroil-lup-20(29)-eno-28-óico.

A substância 9, semelhantemente à substância $\mathbf{8}$, apresentou no espectro de $\mathrm{RMN}{ }^{1} \mathrm{H}$ sinais que caracterizavam a unidade $p$-cumaroíla esterificada com um triterpeno. Foram também observados no espectro de $\mathrm{RMN}{ }^{1} \mathrm{H}$ sinais característicos de substância contendo esqueleto triterpênico. Entretanto, o sinal em $\delta$ 5,20 do hidrogênio olefínico $\mathrm{H}-12$ foi indicativo do esqueleto urseno. No espectro de RMN ${ }^{13} \mathrm{C}$ verificou-se, além dos deslocamentos químicos referentes à unidade $p$-cumaroíla, a presença dos sinais característicos dos carbonos olefínicos dos triterpenos do grupo urseno em $\delta$ 125,4 (C-12) e $\delta 138,1$ (C-13). Da mesma forma que o observado para a substân- 
cia 8, a presença do íon molecular registrado em $\mathrm{m} / \mathrm{z}, 602$ no espectro de massas sugeriu a fórmula molecular $\mathrm{C}_{39} \mathrm{H}_{54} \mathrm{O}_{5}$, corroborando a informação que o triterpeno estava esterificado com o ácido $p$ cumárico. Este espectro mostrou ainda o pico base em $\mathrm{m} / \mathrm{z}$ 147, típico da unidade $p$-cumaroíla. A comparação com os valores de RMN ${ }^{13} \mathrm{C}$ da literatura empregando como modelos o ácido 3- $\beta$-O-trans- $p$ cumaroil-2 $\alpha$-hidroxi-urs-12-en-28-óico e acetato de $\alpha$-amirina permitiu propor a estrutura da substância 9 como sendo o ácido $3 \beta-\mathrm{O}-$ trans-p-cumaroil-urs-12-eno-28-óico.<smiles>CC1CCC2(C(=O)O)CC[C@]3(C)C(=CCC4C5(C)CCC(OC(=O)/C=C/c6ccc(O)cc6)C(C)(C)C5CCC43C)C2C1C</smiles>

O primeiro estudo realizado com o caule de um espécime de $M$. guianensis coletada em Demerara - Georgetown (Guiana) relata o isolamento de 3-oxo-21 $\beta$ - $H$-hop-22(29)-eno (moretenona) e ácido 3 -acetilaleuritólico ${ }^{5}$. Neste reestudo a partir do caule de um espécime coletado na Bahia (Brasil) não foi isolado o ácido 3-acetilaleuritólico e apenas a presença do 3-oxo-21 $\alpha$ - $H$-hop-22(29)-eno ou moretenona, foi coincidente. Entretanto, além de 1, foram isolados $\beta$-sitosterol, lupenona e lupeol e a mistura dos $E$-ferulatos de dodecosila (2), tetracosila (3), hexacosila (4), octacosila (5), triacontila (6), bem como os ácidos 3- $\beta$-acetoxi-lup-20(29)-eno-28-óico (7),


cumaroil-urs-12-eno-28-óico (9). A ocorrência de triterpenos esterificando ácido benzóico e derivados de ácido cinâmico parece ser comum em espécies do gênero Maprounea, tendo sido também registrada em $M$. africana e $M$. membranacea ${ }^{15}$.

O extrato hexânico do caule de Maprounea guianensis não apresentou atividade citotóxica (DL > 1000), enquanto que os extratos clorofórmico e acetato de etila apresentaram $90 \%$ e $100 \%$ de atividade, respectivamente. As substâncias 1-9 apresentaram $\mathrm{DL}_{50}>1000$, mostrando-se inativas. Substâncias que apresentam $\mathrm{LD}_{50}>$ $1000 \mu \mathrm{g} / \mathrm{mL}$ são consideradas inativas e as substâncias que apresen$\operatorname{tam} \mathrm{LD}_{50} \leq 100 \mu \mathrm{g} / \mathrm{mL}$ são muito ativas, comparáveis à camptotecina e ao sulfato de vincristina. As substâncias medianamente ativas são comparáveis ao ácido hipúrico e apresentam $\mathrm{DL}_{50} \geq 100 \mu \mathrm{g} / \mathrm{mL}$, no intervalo entre 100 e $900 \mu \mathrm{g} / \mathrm{mL}^{9}$.

\section{AGRADECIMENTOS}

Os autores agradecem à CAPES e ao CNPq pelas bolsas e auxílio financeiro.

\section{REFERÊNCIAS}

1. http://www.biodiversity.uno.edu/delyt, acessada em Outubro 2002.

2. Calixto, J. B.; Yunes, R. A.; Neto A. S.; Valle, R. M.; Rae, G. A.; Braz. J. Med. Biol. Res. 1984, 17, 313; Syamasundar, K. V.; Singh, B.; Thakur, R. S.; Husain, A.; Kiso, Y; Hikino, H.; J. Ethnopharmacol. 1985, 14, 41; http:/ /www.rain-tree.com/aveloz.htm, acessada em Janeiro 2003.

3. Pengsuparp, T.; Cai, L.; Fong, H. S.; Kinghorn, A. D.; Pezzuto, J. M.; J. Nat. Prod. 1994, 57, 415; Wani, M. C.; Schaumberg, J. P.; Taylor, H. L.; Thompson, J. B.; Wall, M. E.; J. Nat. Prod. 1983, 46, 537.

4. Allem, A. C.; Acta Amazonica 1976, 6, 417; Esser, H. J.; Novon 1999, 9, 32.

5. McLean, S.; Perpickdumont, M.; Reynolds, W. F.; Jacobs, H.; Lachmansing, S. S.; Can. J. Chem. 1987, 65, 2519.

6. Serrano, C.; Ortega, T.; Villlar, A.; Phytother. Res. 1996, 10, 118.

7. Baldé, A. M.; Claeys, M.; Pieters, L. A.; Wray, V.; Vlietinck, A. J.; Phytochemistry 1991, 30, 1024;

8. Correia, S. J.; David, J. P.; David, J. M.; Quim. Nova 2003, 26, 36.

9. David, J. P.; Silva, E. F.; Moura, D. L.; Guedes, M. L. S.; Assunção, R. J.; David, J. M.; Quim. Nova 2001, 24, 730.

10. Wenkert, E.; Baddeley, G. V.; Burfitt, I. R.; Moreno, L. N.; Org. Mag. Resonance 1978, 11, 337; Mahato, S. B.; Kundu, A. P.; Phytochemistry 1994, 37, 1517.

11. Ogunkoya, L.; Phytochemistry 1981, 20, 121.

12. Chávez, J. P.; David, J. M.; Yang, S.; Cordell, G. A.; J. Nat. Prod. 1997, $60,909$.

13. Olea, R. S. G.; Roque, N. F.; Quim. Nova 1990, 13, 278.

14. Lee, S. S.; Shy, S. N.; Liu, K. C. S.; Phytochemistry 1997, 46, 549; Pauletti, P. M.; Araújo, A. R.; Bolzani, V. S; Quim. Nova 2002, 25, 349.

15. Chaudhuri, S. K.; Fullas, F.; Brown, D. M.; Wani, M. C.; Wall, M. E.; Cai, L. N.; Mar, W.; Lee, S. K.; Luo, Y. D.; Zaw, K.; Fong, H. H. S.; Pezzuto, J. M.; Kinghorn, A. D.; J. Nat. Prod. 1995, 58, 1; Beutler, J. A.; Kashman, Y.; Tischler, M.; Cardellina, J. H.; Gray, G. N.; Currens, M. J.; Wall, M. E.; Wani, M. C.; Boyd, M. R.; J. Nat. Prod. 1995, 58, 1039. 